\title{
MACHINE LEARNING FOR THE IMPROVEMENT OF SPRINGBACK MODELLING
}

\author{
Dezelak, M.*; Pahole, I. **; Ficko, M. ${ }^{* *}$ \& Brezocnik, M. ${ }^{* *}$ \\ *Emo-orodjarna d.o.o., Bezigrajska cesta 10, SI-3000 Celje, Slovenia \\ ${ }^{*}$ University of Maribor, Faculty of Mechanical Engineering, Smetanova 17, SI-2000 Maribor, \\ Slovenia \\ E-mail: mihael.dezelak@emo-orodjarna.si
}

\begin{abstract}
:
New demands in the automotive industry have led to an increase in the use of Advanced High-Strength sheet metal materials. However, higher values of strength are usually achieved at the expense of reduced formability and increased sensitivity of the springback. Today, springback is one of the more important factors that influence the quality of sheet metal forming products. During the forming process, sheet metal undergoes a complicated deformation history, which is why the accurate prediction of the springback level can be very difficult. Today, a good compromise between the finite element method (FEM) simulation and the real stamping process can be achieved, but there is still limited reliability of the FEM springback prediction. In this paper, the machine learning $(\mathrm{ML})$ approach was used to update the FEM for springback modelling. Combined models are tuned to better reflect the measured experimental data.
\end{abstract}

Key Words: Sheet Metal Forming, Springback, Machine Learning

\section{INTRODUCTION}

Springback of sheet metal products is a very complex problem. It is the result of the stress state in the material following the forming process and means a change of shape in the sheet metal forming product after the withdrawal of the forming forces. Steel sheets with high strength and aluminium alloys are more sensitive to the springback effect due to a greater degree of elastic deformation than conventional mild steels.

A comprehensive examination of the current estimation of the springback of sheet metal after forming is shown in the work [1]. Analytical, experimental and numerical approaches are introduced in detail. Analytical solutions in their completeness are only valid for simple ideal cases, but it also provides advanced understanding of the relations between some of the material and the process parameters that increase or decrease the level of springback [2].

Numerical simulations are a kind of approximation for the real behaviour of sheet metal during processing. By modelling the FEM (finite element method) the physical structure of the sheet metal and tools is converted into a mathematical model for the solution to use the numerical procedure, in particular, the modelling material properties of the sheet and the contact and friction conditions of the tool depend on the reliability of the forming process with computer simulations. For the conversion of sheet metal material to a numerical model a few material models were developed. The advantages and disadvantages of the different material models and the standard experiments to determine their parameters are presented in the work [3].

It is necessary to know and understand the influence of the numerical parameters on the simulation results for the successful application of the FEM to predict springback. Full reviews of the numerical simulation of sheet metal forming are presented by many authors $[4,5]$. These are guidelines for the determination of material models and numerical simulation parameters for different materials, followed by a comparison with the results of 
real experiments [6]. Knowledge in the evaluation of the simulation forming process of conventional mild steel using the FEM is at a relatively high-level [7], more difficulties are encountered in the FEM simulations of new, advanced materials [8]. In the work [9] there is presented the effect of a variation of the technological and numerical parameters on the springback results of numerical simulations of simple examples of formed sheet metal.

It is impossible to completely overcome springback but it can be limited and monitored by setting the optimal forming parameters. The product must, after completing the operations, be within certain tolerance limits, it is the final stage of tool-making that usually requires the lengthy manual geometry optimization of the punch, dies and drawbeads, and also the optimization of the holding force [10] can play an important role in forming product stability. Potential solutions to this problem are the methods of springback compensation to simulate springback at the planning stage of the forming process [11]. There are several well developed iterative springback compensation methods whose efficiency is conditional on the accuracy of the springback simulation.

\section{BACKGROUND OF THE RESEARCH}

\subsection{Machine Learning for Springback Prediction}

Machine Learning (ML) is a field of information science with an interdisciplinary character. Using ML methods, branches of computational intelligence, have flourished in recent years in many areas, as computers become more powerful and accessible to a wider circle of researchers and users in industry. Methods of $M L$ are useful tools in predicting springback in the sheet metal forming process. In the paper [12] there is presented the springback prediction using the method of an artificial neural network, the training and evaluation process is carried out based on the results of computer simulations using the FEM. Artificial neural network analyses of air bending are also presented in the paper [13], the emphasis is on analyzing the impact of using a different amount of data for training the network on the quality of the result. In [14], genetic algorithms were used to optimize the weights of the neural networks in order to reduce discrepancies between the predicted punch radius by the neural network and the measured value. We have seen in combination with the FEM for springback prediction with evolutionary algorithms. In paper [15] the latter was performed to evaluate the optimal process parameters. The results have been stated of computer simulations of a FEM for the derogation from the real physical system behaviour. The reasons for this are the specific errors and approximation in modelling the real process, as well as the limitations of computer capacity. The paper [16] presents an approach using the method of data mining to correct results of FEM springback simulations and minimizes the deviation of the results of FEM simulations of the experimental values.

Experience shows no single ML scheme is appropriate for all data mining problems. The universal learner is an idealistic vision. The choice of $M L$ approach depends on the nature of the problem that we are researching. Regression methods are appropriate for the update of the results of FEM simulations. The task of the regression predictor, the pattern described by a set of attributes sets the value of the dependent regression. The learning algorithm must therefore be in the numerous samples with known values of the dependent variable to calculate a continuous function, which can be used to determine the value of the regression of variable new cases. The regression predicator can be separated according to the type of presentation of the regression function.

\subsection{Draw/bend test}

The basis of our research was an experiment called the draw/bend test. This is a simplified method of deep drawing process, where sheet metal is pulled over the radius (Figure 1). 


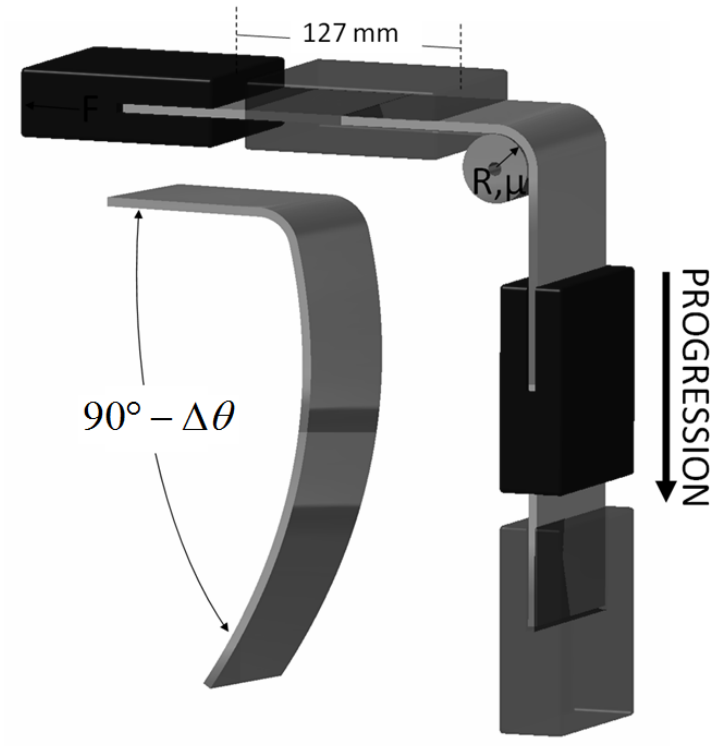

Figure1: Draw/bend test [17].

After the experiment progression, springback occurred. It is the result of flexible angular changes and sidewall curl. Their sum is expressed in $\Delta \theta$ corner. For three different materials, three parameters such as tool radius, restraint force and friction were analyzed. Details of the experiment and the results were summarized by source [17].

\section{METHODS}

\subsection{FEM Springback Modelling}

The FEM springback prediction results take their source from the quality of the performed forming simulation. A number of numerical parameters in each simulation step can have a significant impact on the final forecast changes in geometry after the withdrawal of the forming forces, and the latter were not varied using the default values, and we focussed only on technological parameters. For the modelling of the above-mentioned experiment we used the program PamStamp by which we try to make the best model of the real drawing process of sheet metal through a radius tool.

The initial blank mesh size was $5 \mathrm{~mm}$ and during the simulation refined by factor 3 . Accurate contact and different friction coefficients were set (depending on the experiment case). The surfaces of the tools were considered as rigid bodies. Shell elements were used with the Gauss thickness integration rule and with five integration points through the thickness. Material parameters were taken from reference [17]. The simulation results using the FEM are presented in the next section.

\subsection{Machine Learning for updating FEM results}

For improving our FEM springback results a Weka workbench was used [18]. The Weka workbench is a collection of ML algorithms and data preprocessing tools. It includes methods for all the standard data mining problems: regression, classification, clustering, association rule mining, and attribute selection. We used classification methods under the function category, because the "new knowledge" can be written down as a mathematical equation in a reasonably natural way. In our case, the ML system based on experiment and simulation draw/bend test, six different algorithms were used: Linear Regression, Isotonic Regression, Least Medium Square, SMO, Gaussian Processes and Multilayer Perceptron [18]. The first three methods are linear regression methods. Linear Regression performs standard leastsquares multiple linear regression and can optionally perform different attribute solution 
rules. Least Medium Square is a robust linear regression method that minimizes the median of the squares of divergences from the regression line. Isotonic Regression implements the method for learning an isotonic regression function based on the pair-adjacent violators approach. SMO implements the sequential minimal optimization algorithm for training a support vector classifier, using kernel functions such as polynominal or Gaussian kernels. Missing values are replaced globally; nominal attributes are transformed into binary ones. Gaussian Processes is a method that implements the Bayesian Gaussian process technique for nonlinear regression. Multilayer Perceptron is a neural network that trains using backpropagation. All ML methods used for springback prediction in this paper are described in detail in scientific literature $[18,19,20]$.

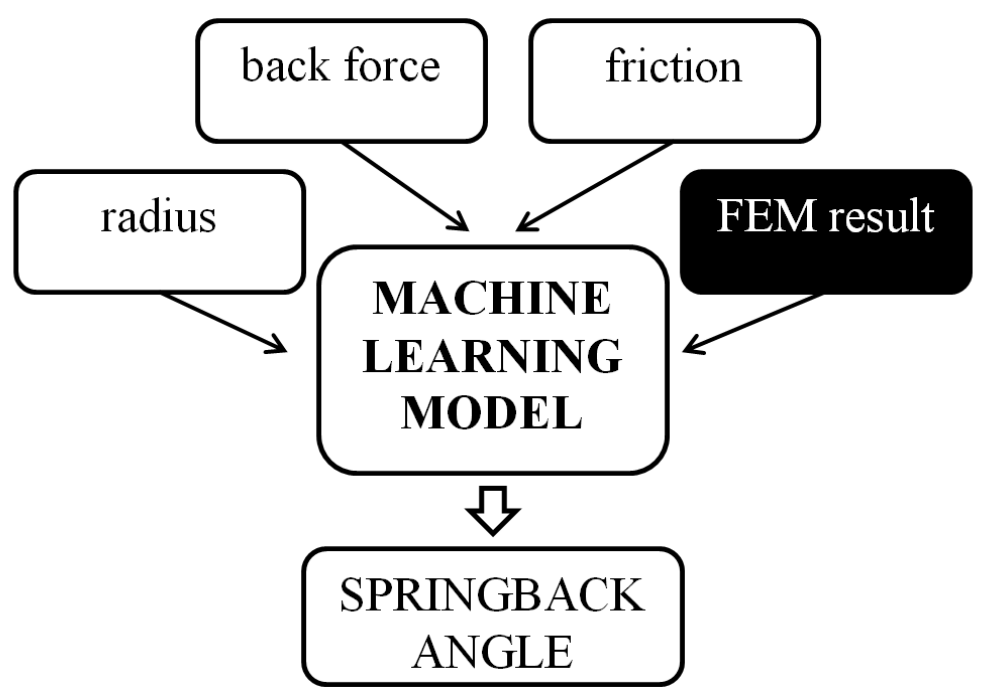

Figure 2: Machine Learning scheme.

The attributes (inputs) for each case of sheet metal material were radius, normalized holding force or back force, the coefficient of friction and the result of the FEM simulation (Figure 2). The regression variable as a measured angle value in the experiment we assume to be absolutely correct. Furthermore, after the partial processing of data sets for each material, a common machine learning model was investigated and new attributes - material parameters were used (sheet thickness, yield stress, factor of normal anisotropy and elastic module Figure 3).

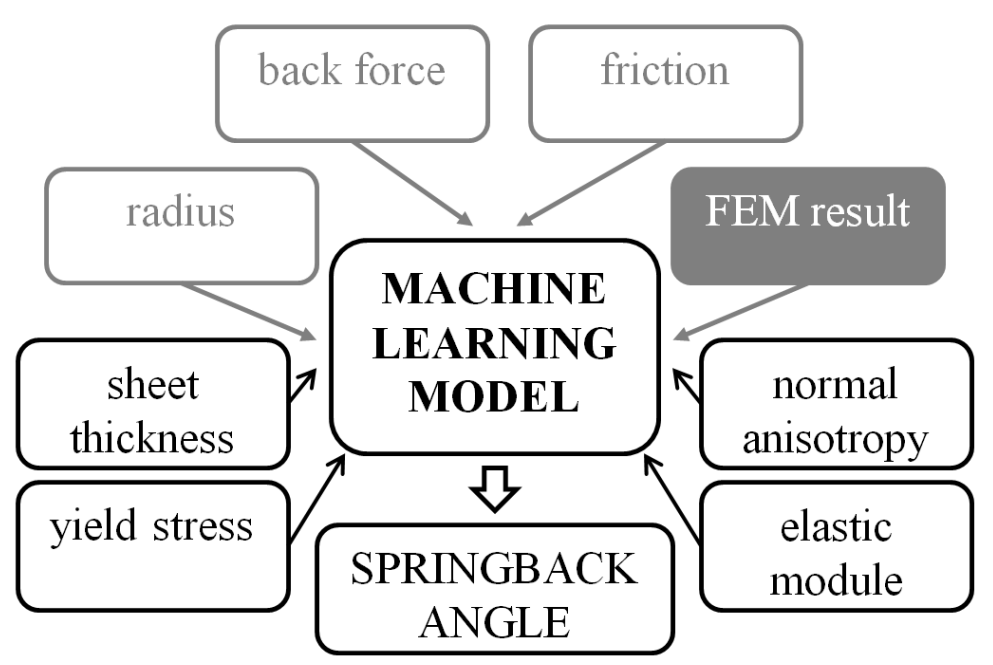

Figure 3: Machine Learning scheme - common model. 
From the all data sets it is necessary to define data sets on which the model learns, and strings, on which the method is tested. Because of the limited amount of sets for training and testing, the cross-validation method was chosen. In each step, the method of testing used a certain proportion of data sets (in our case 10\%), the rest is intended to facilitate learning. The procedure was repeated 10 times, so that all the strings were used at least once, both for learning as well as for testing. The total error of the method is defined as the average error of all ten steps. To evaluate the accuracy of the methods' models, we used the criterion of the correlation coefficient. The correlation coefficient measures the statistical correlation between the predicted values and the actual values. The correlation coefficient ranges from 1 for perfectly correlated results, through 0 when there is no correlation, to -1 when the results are perfectly correlated negatively [18]. Of course, negative values should not occur for the reasonable prediction methods.

The reliability of the ML methods depends on many factors. The problem can be defined by more attributes and more accurate output values can be expected. A wide set of data also increases the efficiency of the ML. In our case there was a complex problem of the springback of the sheet metal available in a relatively small number of attributes and also a narrow learning set. Both are expressed within the somewhat limited precision of the ML.

\section{RESULTS AND DISCUSSION}

Figure 4 demonstrates the experimental [17] springback results and the results of FEM simulations for the HSLA material. The FEM simulation results deviate slightly from the experimental values. The good correlation between the FEM simulation results and the experiment is also expressed in $\mathrm{ML}$, since the presence of attribute FEM simulation result significantly improves the correlation model of the $\mathrm{ML}$ algorithms presented overall (Figure 5). Compared to the solely FEM method an even higher correlation with the FEM combined Linear Regression and Multilayer Perceptron method was reached.

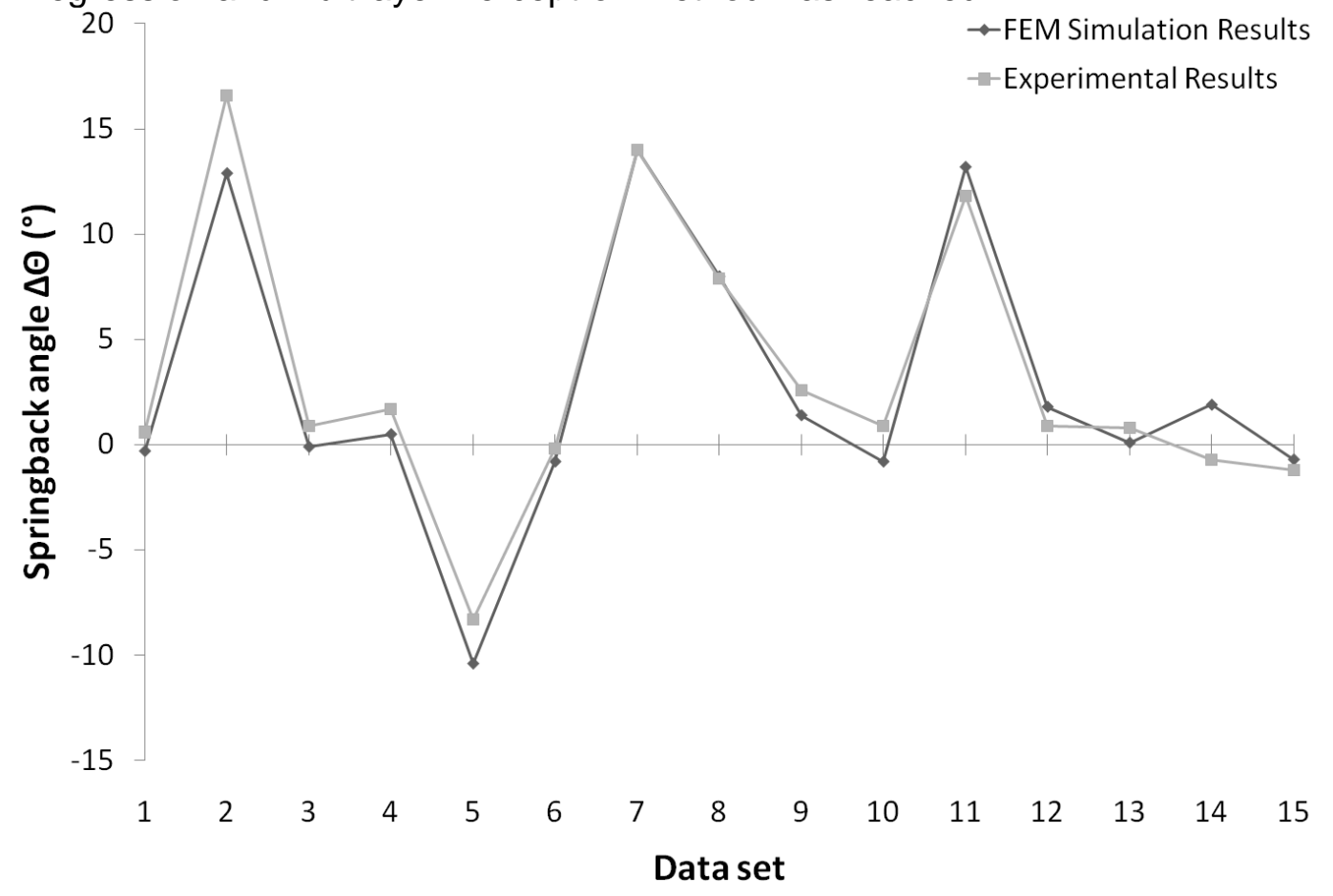

Figure 4: HSLA - FEM and experimental [17] results. 


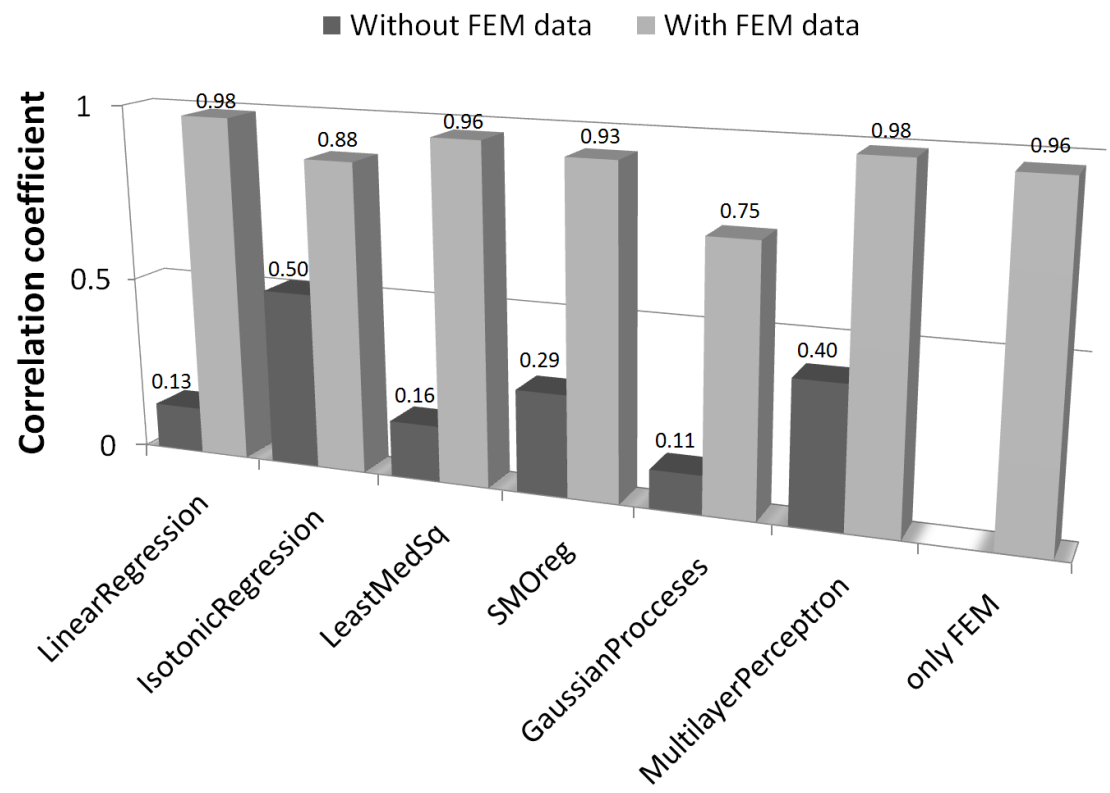

Figure 5: HSLA, correlation coefficients of ML models.

Figure 6 presents the FEM simulation and experimental [17] results of springback for 6022T4 material. For some examples, the springback results are comparable, but we see some substantial variations that reduce the data quality for ML. Causes for the deviations could be related to the number of approximations in the numerical models, since we assumed that the experimental results were accurate. In normal procedure of ML, we usually have broad learning data sets with large variations and we can simply cross the most divergent out of the learning sets. Compared to the solely FEM results, several solely ML methods perform an even higher correlation. However, combined models in this case do not normally improve the reliability of the springback prediction. There is just one exception; Multilayer Perceptron (Figure 7).

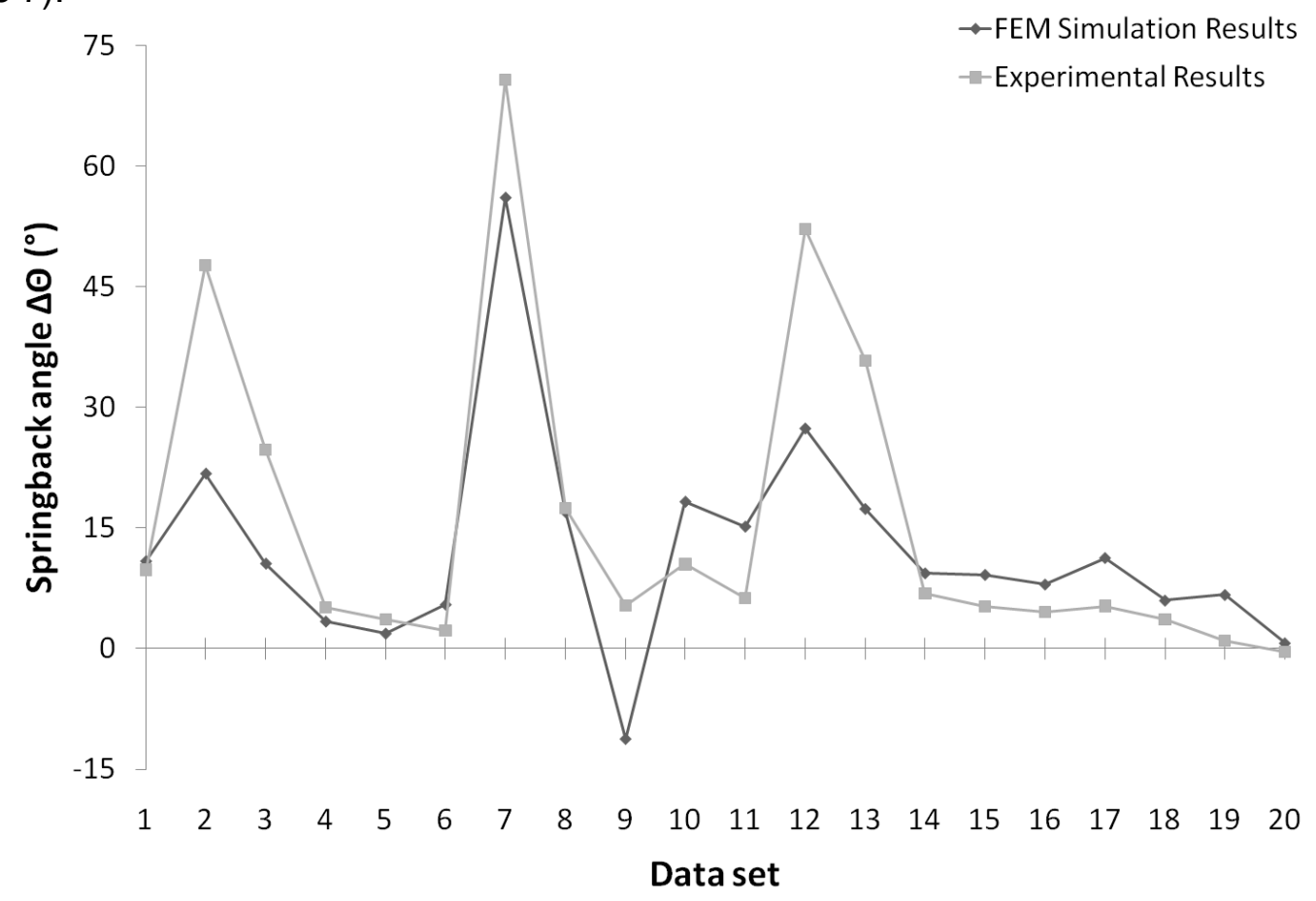

Figure 6: 6022-T4 - FEM and experimental [17] results. 
Figure 8 presents the results of FEM simulations and experiments [17] for the material DQSK, representative of mild steel sheet. In these mild steel sheets the springback problem is not as significant as in the previous two cases. This can also be seen from our results of FEM simulations and experiments. But at low absolute values, springback shows a relatively large deviation in the results of FEM simulations and experiments, which reduces the quality of the learning sets of $\mathrm{ML}$ (Figure 9). If we exclude the three most divergent data sets $(7,8$, 14 - Figure 8), the ML prediction model is essentially improved for both, with and without the FEM simulation results (Figure 10).

Without FEM data With FEM data

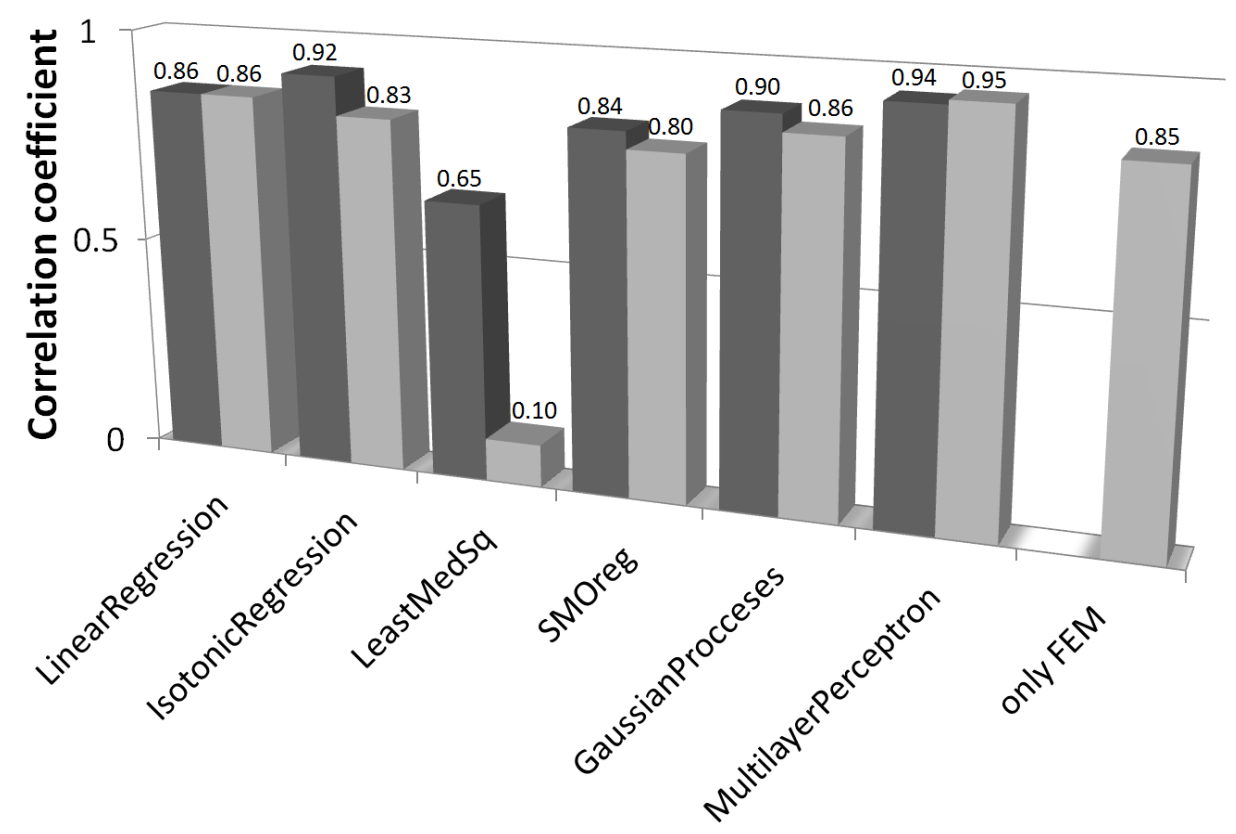

Figure 7: 6022-T4, correlation coefficients of ML models.

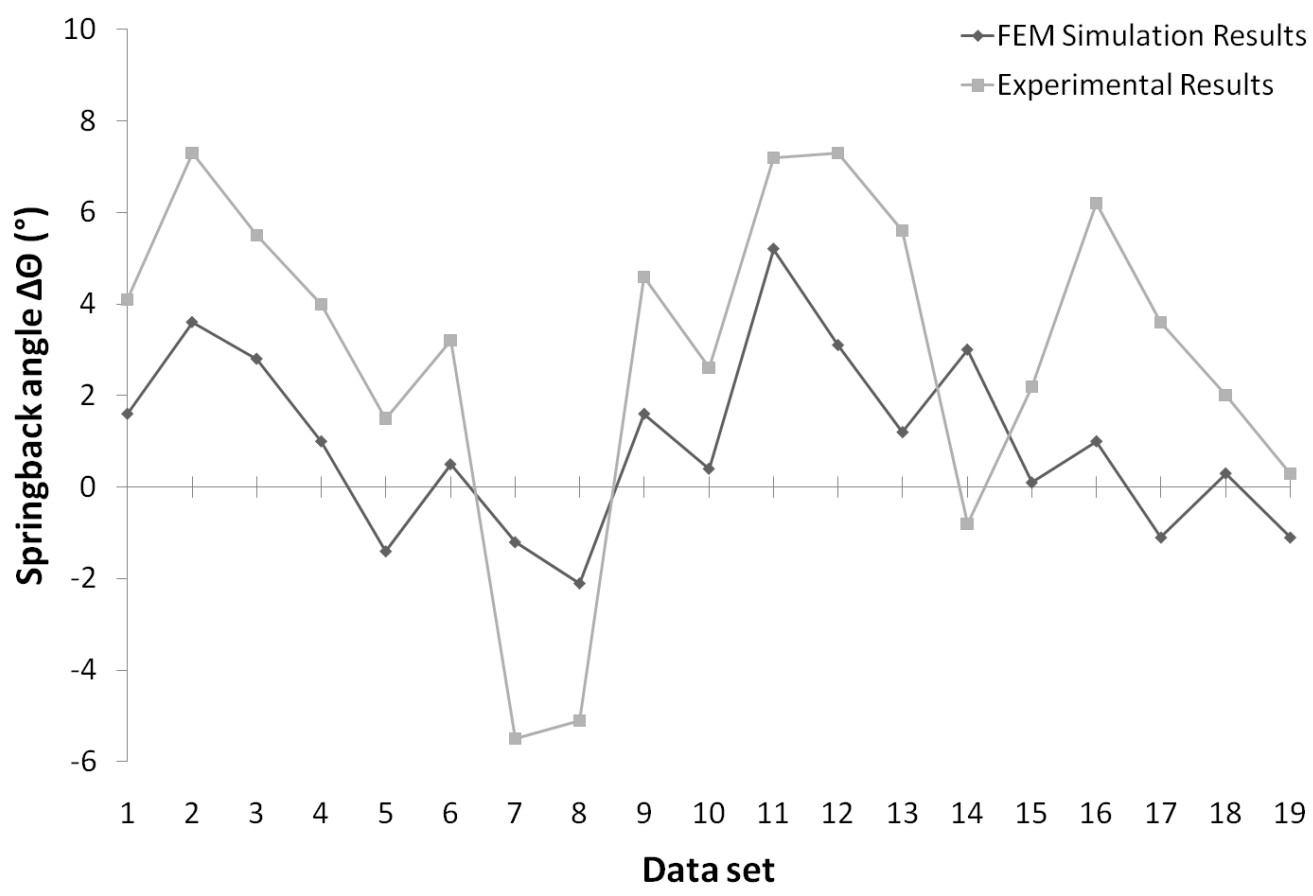

Figure 8: DQSK - FEM and experimental [17] results. 


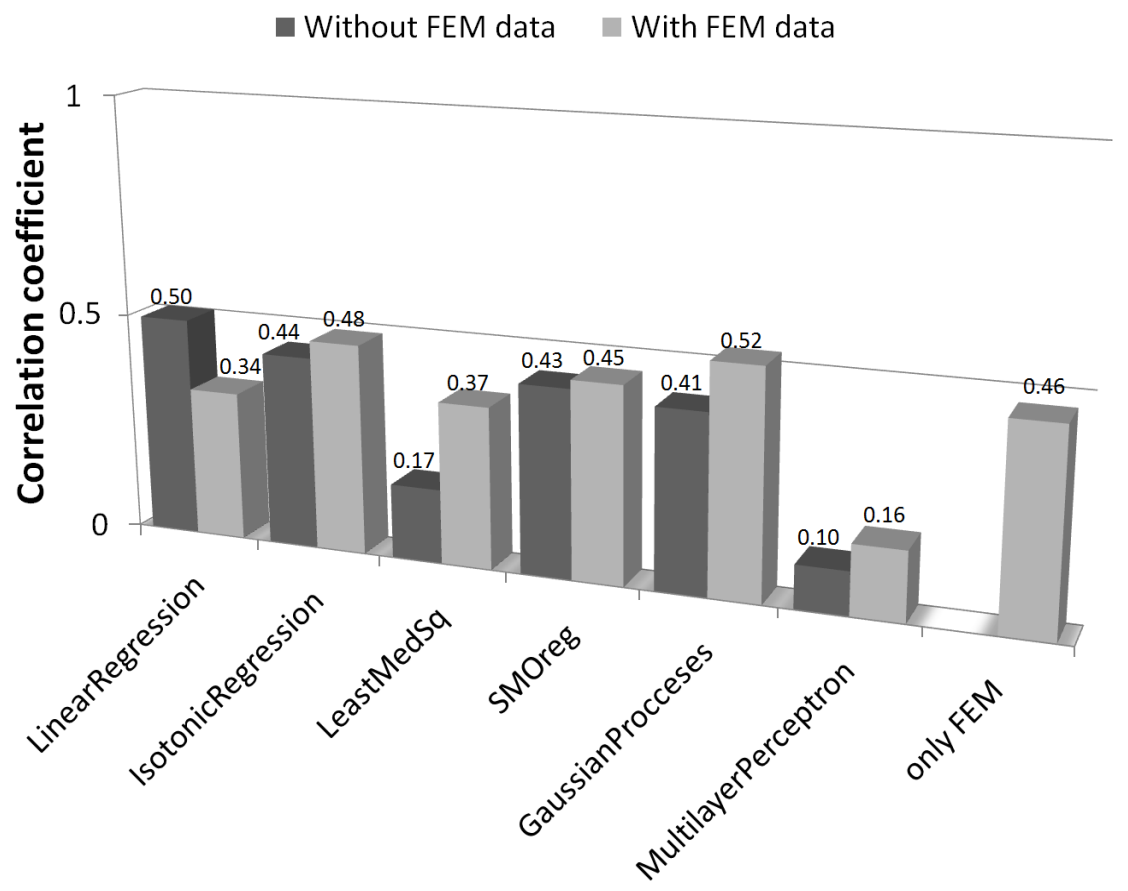

Figure 9: DQSK, correlation coefficients of ML models.

Without FEM data correction

With FEM data correction

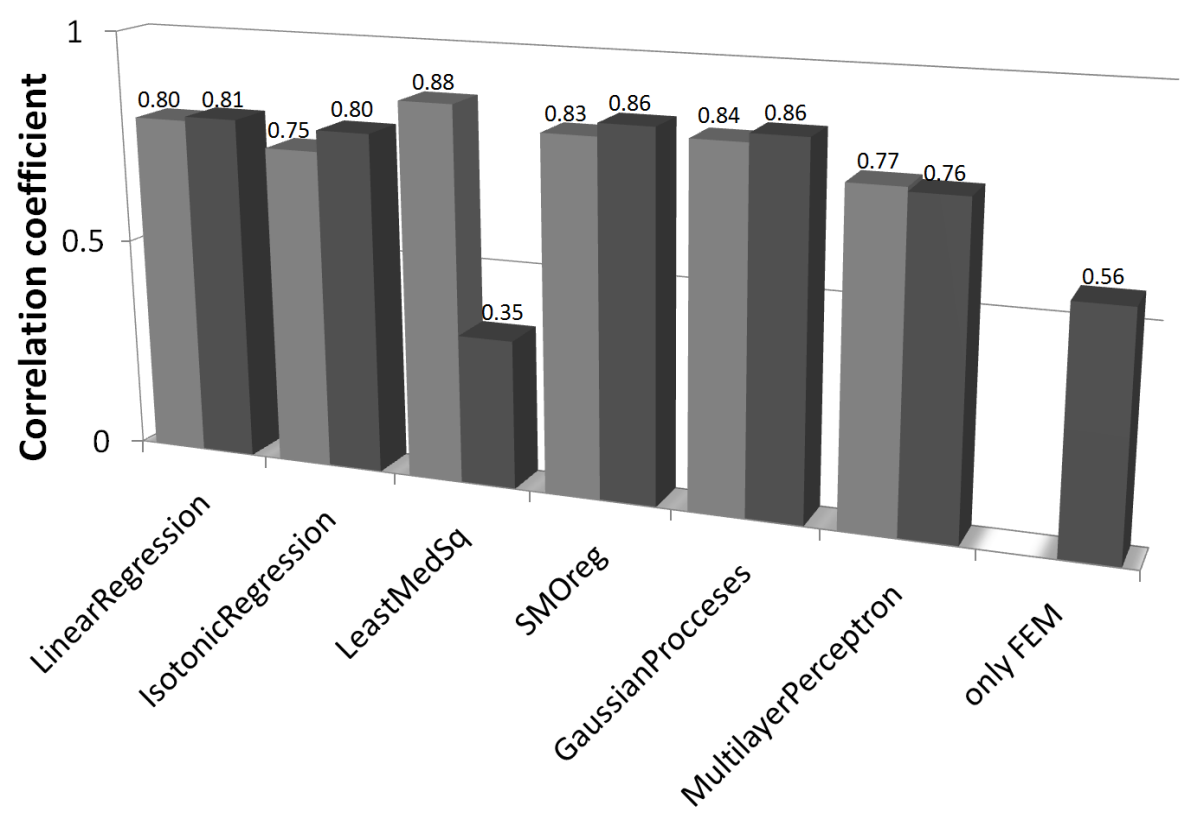

Figure10: DQSK, correlation coefficients of ML models for corrected data sets.

Furthermore, a common ML model was created. More attributes (four material parameters) and data sets were available for this procedure and despite the consideration of all data sets (inaccurate data were also used) we obtained a pretty well learned model. This can be seen in Figure 11, where correlation coefficients for all six ML algorithms are presented. If we take a look at the results of the correlation coefficients we can see that the last three tested methods (SMO, Gaussian Processes and Multilayer Perceptron) are more suitable for springback prediction. One of the reasons is definitely in the nonlinearity of the complex springback phenomenon and these methods are able to model this 
kind of phenomena. However, combination with the FEM raises the correlation coefficient to a higher level compared to the solely FEM method (Figure 10).

\section{Without FEM data With FEM data}

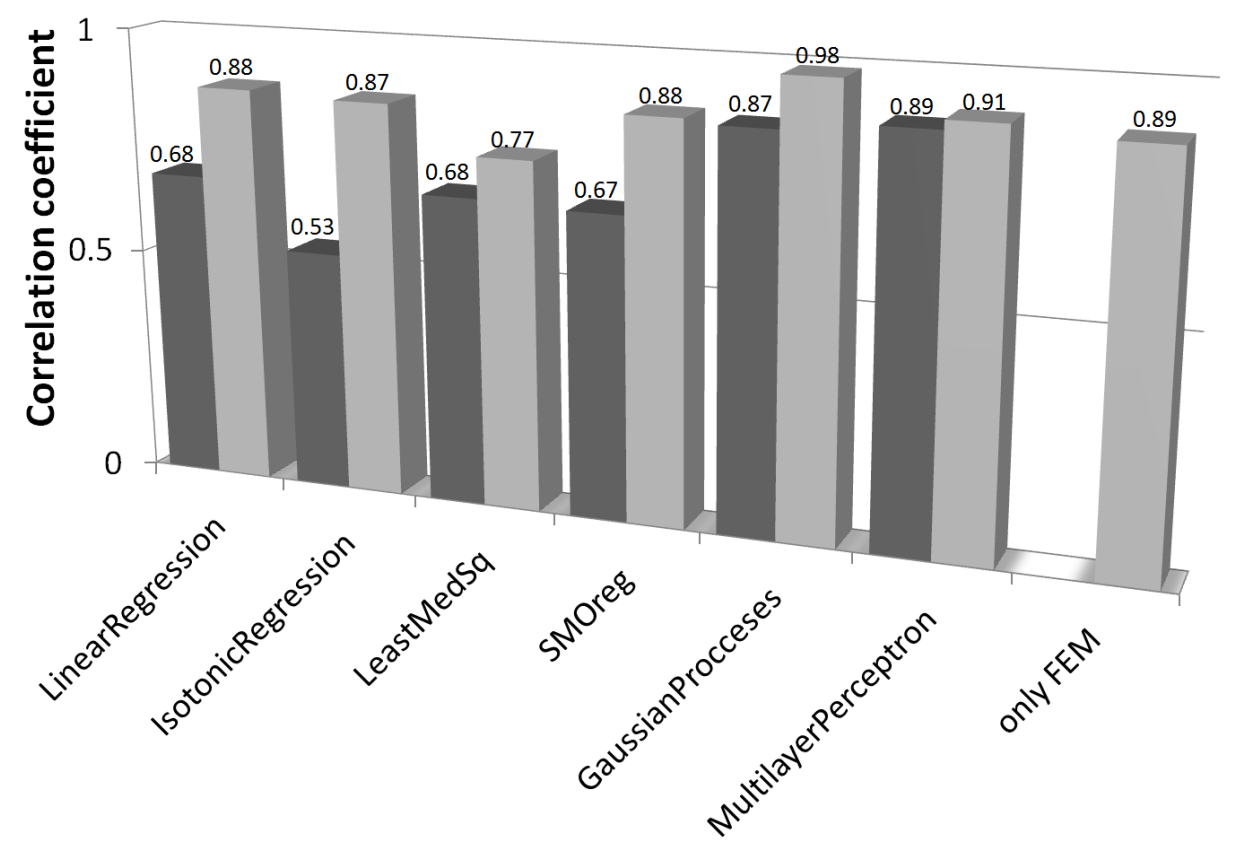

Figure 11: Common model correlation coefficients of ML.

\section{CONCLUSION}

This paper presents a case of combining two methods of predicting springback: the finite element method (FEM) and machine learning (ML). The FEM simulation results were compared to the experimental results [17] and learning sets of ML were created. ML for each material case was performed by six ML methods and the correlation between the actual and the predicted values was investigated. Finally, a common model for all three materials was made and tested. It was shown that the combination of both presented methods with adequately precise results of the FEM simulations usually allows a more accurate springback prediction compared to the solely FEM method.

In the presented examples, only relatively well predictable parameters (attributes) were used for springback prediction. However, we are aware of the fact many springback problems can arise because of unknown parameters, such as material tolerances, producers of the sheet metal, date of production... These are all potential attributes for ML modelling and they could improve springback prediction. With ML algorithms there is the possibility of recording all successful solutions of springback reduction and compensation, and to use them on new more or less similar cases.

\section{ACKNOWLEDGEMENTS}

"Operation part financed by European Union, European Social Fund."

\section{REFERENCES}

[1] Burchitz, I. A., (2008). Improvement of springback prediction in sheet metal forming, Thesis. Faculty of Engineering Technology, University of Twente

[2] Yi, H. K., Kim, D. W., Van Tyne, C. J., Moon, Y. H., (2008). Analytical prediction of springback based on residual differential strain during sheet metal bending, Journal of Mechanical Engineering Science, vol. 222, no. 2, p. 119-129

[3] Hermen, P., (2001). Sheet material characterization by multi-axial experiments, Thesis. Faculty of Engineering Technology, University of Twente 
[4] Meinders, T., (2000). Developments in numerical simulations of the real-life deep drawing process, Thesis. Faculty of Engineering Technology, University of Twente

[5] Stepišnik, A., Kuzman, K., (2003). Knowledge, experience and numerical sheet metal process simulations - a basis for fast and reliable preparation of an offer. International Deep Drawing Research Group, Conference Proceedings, p. 225-231

[6] Pahole, I., Puc, M., Vaupotič, B., Balič, J., (2006). Comparison of technology of forming the sheet metal by numerical simulations, Journal of Achievements in Materials and Manufacturing Engineering, vol. 18, no. 1-2, p. 403-406

[7] Pahole, I., Bonifarti, S., Ficko, M., Vaupotič, B., Kovačič, S., Balič, J. (2006). Bending of sheet metal of complicated shapes (for $90^{\circ}$ angle and more) in combined tools, Journal of Achievements in Materials and Manufacturing Engineering, vol. 16, no. 1-2, p. 88-93

[8] Deželak, M., Stepišnik, A. (2010). Numerical Simulation of sheet metal forming product of AHSS in cold (in Slovene). Industrijski forum IRT, p. 153-158

[9] Deželak, M., Pahole, I., Stepišnik, A., Stepišnik, S., Gusel, L. (2010). The impact of technological and numerical parameters on springback prediction of AHSS after forming (in Slovene), Orodjarstvo in strojegradnja 2010, p. 147-153

[10] Volk, M., Deželak, M., Nardin, B., Stepišnik, S. (2011). Prediction of the Spring-back Calculated with Numerical Simulations for the Household Industry, Numisheet 2011, Seoul, Korea, Conference Proceedings, p. 1078-1085

[11] Meinders, T., Burchitz, I.A., Bonte, M.H.A., Lingbeek, R.A. (2008). Numerical product design: Springback prediction, compensation and optimization. International Journal of Machine Tools \& Manufacture, vol. 48, p. 499-514

[12] Kazan, R., Firat, M., Tiryaki, A.E. (2009). Prediction of springback in wipe-bending process of sheet metal using a neural network, Materials \& design, vol. 30, no. 2, p. 418-423

[13] Forcellese, A., Gabrielli, F., Ruffini, R. (1998). Effect of the training set size on springback control by a neural network in an air bending process. Journal of Materials Processing Technology, vol. 80-81, p. 493-500

[14] Fu, Z., Mo, J., Chen, L., Chen, W. (2010). Using genetic algorithm - back propagation neural network prediction and finite element model simulation to optimize the process of the multiplestep incremental air-bending forming of sheet metal, Materials \& design, vol. 31, no. 1, p. 267277

[15] Liew, K.M., Tan, H., Ray, T., Tan, M.J. (2002). Optimal process design of sheet metal forming for minimum springback via an integrated neural network evolutionary algorithm, Structural and Multidisciplinary Optimization, vol. 26, no. 3-4, p. 284-294

[16] Xu, J., Wang, X., Zhang, P., Wu, Y. (2003). Springback Prediction in Sheet Metal Forming Combined Finite Element Method with the Date Mining Technique. School of Mechanical Engineering, Shanghai University

[17] Carden,W.D., Geng, L.M., Matlock, D.K., Wagoner, R.H. (2002). Measurement of springback. International Journal of Mechanical Sciences, vol. 44, p. 79-101.

[18] Witten, I. H., Frank, E., Hall, M. A. (2011). Data Mining, Practical Machine Learning Tools and Techniques, Third Edition, Elsevier

[19] Mitchell, T.M. (1997). Machine Learning. McGraw-Hill

[20] Konenko, I. (2005). Machine Learning (in Slovene). Zal. FE in FRI, Ljubljana 